\title{
Gujarati Text - To - Speech System
}

\author{
Samyak Bhuta and S. Rama Mohan
}

The M S University of Baroda, Vadodara, Gujarat, India

The need for text-to-speech systems in various languages is obvious with the current fast paced technology development in information and communication technology. Keeping the Gujarati language, intimately used by 55 odd million people in India and abroad, abreast with technology development is not just logical but heartfelt.

The Gujarati Text-to-speech system should be an apparatus that should take arbitrary Gujarati text as input and should produce the equivalent speech sounds keeping the phonetic and prosodic concerns intact. The quality of the generated sound output should be determined by two parameters viz., intelligibility and naturalness.

With these objectives in mind, a Gujarati Text-to-Speech system has been developed at the Resource Center for Indian Language Technologies for Gujarati at The M. S. University of Baroda, Vadodara with financial support from the Ministry of Communications and Information Technology of Government of India.

The approach used in synthesizing the speech is that of concatenation of segments of pre-recorded speech sound. The system's core engine has been developed to operate with any type of concatenation unit i.e., partneme, diphone, disyllable etc. ( or mix of them ) to form a desired output. It is to be noted that the speech engine has been developed from the ground level instead of borrowing an existing engine, for the sake of greater flexibility that it would provide.

The engine is designed to take the speech input in International Phonetic Alphabet. This also helps in achieving the separation of concerns with respect to the language independent aspects of the system from those of the language dependent parts. In our experiments we have found that working on the disyllable level as concatenation units provides better results than working with the partneme or diphoneme as the concatenation units. This happens since most of the concatenations occur at the midpoints of the vowel sounds.

Results of the output sound from the Gujarati TTS system are encouraging, being both intelligible and natural to a good extent. The system also takes care of common abbreviations and pronouncing numbers by pre-processing. But the database of disyllables is still short of being complete. Efforts to complete the data base are continuing.

Many improvements can be brought to the system in addition to completing the data base of disyllables. These include making system more efficient from computational point of view. Another direction in which the quality of synthesized speech can be significantly improved is the introduction of prosodic inputs that could be garnered by studying the Gujarati phonology. 